Zur Charakteristik der aromatischen $\beta$-Hydantoine ist noch hinzuzufügen, dass dieselben mit Alkalien in Wasser leichtlösliche, in überschüssiger Lauge und in Alkohol schwerlösliche, aus Alkohol gut krystallisierende Verbindungen geben, welche als Salze der betreffenden Hydantoinsäuren von der Formel $\mathrm{NH}_{2} \mathrm{CONRCH}_{2} \mathrm{COOH}$ anzusehen sind.

Durch Zersetzung mit Säuren liefern diese Salze nicht die freien Hydantoinsäuren, sondern wieder die gewissermassen als Anhydride der Hydantoinsäuren zu betrachtenden $\beta$-Hydantoine.

Durch Umsetzung der Kaliumsalze der Hydantoinsäuren mit anderen Salzen erhält man leicht die übrigen, in Wasser entweder schwer oder meist unlöslichen Salze, wie z. B. Baryum-, Calcium-, Silber- und andere Salze.

Die Analyse des

\title{
p-Aethoxylphenylhydantoinsauren Kaliums
}

ergab folgende Zahlen:

0,3189 Substanz gaben mit $\mathrm{H}_{9} \mathrm{SO}_{4}$ geglüht $0,1039 \mathrm{~K}_{\mathbf{q}} \mathrm{SO}_{4}=0,04404 \mathrm{Ka}$ $=13,81 \% \mathrm{Ka}$.

Die Formel $\mathrm{C}_{11} \mathrm{H}_{12} \mathrm{~N}_{2} \mathrm{O}_{8} \mathrm{KOH}$ verlangt 14,13\% Ka.

Weitere Salze der Hydantoinsäuren wurden nicht analysiert, es unterliegt aber keinem Zweifel, dass dieselben analoge Zusammensetzung besitzen, wie das untersuchte.

Da die Hydantoine nur schwach sauren Charakter besitzen, so reagieren die Salze der Alkalien stark alkalisch.

\section{Ueber die Einwirkung von Phenylhydrazin auf Chloracetylharnstoffe und Chloracetylurethan.}

Von G. Frerichs und H. Beckurts.

Vom Phenylhydrazin leiten sich bekanntlich zwei verschiedene Phenylhydrazidoessigsäuren ab, und zwar eine symmetrische und eine

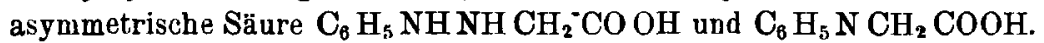<smiles>NI</smiles>

Derivate beider Säuren sind erhalten worden aus Chloressigsäurederivaten und Phenylhydrazin, und zwar scheinen sich die symmetrischen Verbindungen dann zu bilden, wenn der Rest der Chloressigsäure mit . einem Radikal saurer Natur verbunden ist. So erhielt A. Reissert ${ }^{1}$ )

1) Ber. 28,1230 . 
aus Chloressigsäureäthylester und Phenylhydrazin den symmetrischen Phenylhydrazidoessigsäureäthylester $\quad \mathrm{C}_{6} \mathrm{H}_{5} \mathrm{NH} \mathrm{NH} \mathrm{CH}_{2} \mathrm{CO}_{\mathrm{OC}_{2}} \mathrm{H}_{5}$. Liegt dagegen eine Verbindung der Chloressigsäure mit einem Radika! basischer Natur vor, so scheinen sich nur die asymmetrischen Derivate zu bilden. So haben Rupe und Heberlein aus Chloracetanilid ${ }^{1}$ ), ferner aus Chloracetamid ${ }^{2}$ ), sowie aus Chloracetdimethyl-p-phenylendiamin ) durch Einwirkung von Phenylhydrazin die Verbindungen de asymmetrischen Phenylhydrazidoessigsäure dargestellt. Wir haben nuu das Phenylhydrazin auf Chloracetylderivate verschiedener Harnstoffe und des Aethylurethanes einwirken lassen und gefunden, dass in diesen Fällen nicht nur Derivate der einen oder der anderen Phenylhydrazidoessigsäure entstehen, sondern dass die beiden angedeuteten Reaktionen nebeneinander verlaufen, dass aber auch noch sekundäre Reaktionen auftreten, sodass wir aus den Chloracetylharnstoffen jedesmal drei, aus dem Chloracetylurethan zwei verschiedene Körper erhielten. Zur Darstellung und Trennung der verschiedenen Verbindungen erwies sich nach einigen Versuchen tolgendes Verfahren als praktisch:

$20 \mathrm{~g}$ Harnstoffderivat (Chloracetylharnstoff, Methyl-, Phenyl-, p-Aethoxylphenylharnstoff) wurden mit der entsprechenden Menge (2 Mol.) Phenylhydrazin und etwa $200 \mathrm{ccm}$ Alkohol auf dem Dampfbade am Rückflusskühler einige Stunden erhitzt, und die entstandene, stets intensiv gelbrot gefärbte Flüssigkeit erkalten lassen. Die reichlich sich ausscheidenden Krystalle wurden abgesogen und zunächst mit Alkohol, dann mit warmem Wasser, zur Entfernung des ebenfalls mit auskrystallisierten salzsauren Phenylhydrazins, ausgewaschen. Die auf diese Weise erhaltenen gelben Krystalle wurden mit verdünnter Salzsäure und $4-500 \mathrm{ccm}$ Wasser erwärmt, und der hierin unlösliche Anteil abfiltriert und aus Alkohol umkrystallisiert. Die so erhaltenen Körper sollen im folgenden jedesmal als I. Produkt bezeichnet werden.

Die filtrierte salzsaure Lösung wurde noch warm mit Ammoniak übersättigt, wodurch in jedem Falle ein weisser flockiger Niederschlag entstand. Derselbe wurde zur Reinigung mit Wasser gewaschen und aus Alkohol umkrystallisiert; II. Produkt.

Die zuerst erhaltenen, vom Reaktionsprodukt abfiltrierten, alkoholischen, gelbroten Mutterlaugen enthielten jedesmal noch einen dritten gelb gefärbten Körper, welcher sich auf Zusatz von viel salzsäurehaltigem Wasser zu der etwas konzentrierten Mutterlauge zunächst emulsionsartig, auf Zusatz von Kochsalz als flockiger Niederschlag

1) Ber. 28, 1717,

2) Ber. 29, R. 622.

B) Ber. 30, 1101. 
abschied. Diese Art Körper konnten wir nicht in krystallinischem Zustande erhalten, auch scheint eine völlige Reinigung dieser Verbindungen mit Schwierigkeiten verknüpft zu sein, was einige Analysen vermuten liessen. Wir haben diese Körper deshalb vorläufig noch nicht näher untersucht, werden aber später wieder darauf zurückkommen.

Aus Chloracetylurethan und Phenylhydrazin erhielten wir auf die oben erläuterte Weise nur zwei Verbindungen, einen in salzsäurehaltigem Wasser unlöslichen, krystallinischen und einen amorphen gelben Körper. Letzteren haben wir ebenfalls vorläufig noch nicht näher untersucht,

Die als II. Produkt bezeichneten Derivate der Harnstoffe besassen alle, wie die Analyse ergab, die Zusammensetzung der Phenylhydrazidoacetylderivate der verschiedenen Harnstoffe und zwar zeigte die nähere Untersuchung, dass diese Körper Derivate der asymmetrischen Säure von folgender Formel darstellen:<smiles>[R]NC(=O)NC(=O)Nc1ccccc1</smiles>

Es geht dieses daraus hervor, dass diese Körper mit Aldehyden und Ketonen genau so reagieren wie das Phenylhydrazin, ausserdem wurde durch Verseifung daraus eine Säure erhalten, welche unzweifelhaft die asymmetrische Säure darstellte. Auf alkalische Kupferlösung wirken diese Verbindungen schon bei gelindem Erwärmen stark reduzierend.

\section{a-Phenylhydrazidoacetylharnstoff}<smiles>NCC(=O)NC(N)=O</smiles>

Aus Chloracetylharnstoff und Phenylhydrazin dargestellt, bildet derselbe glänzende, farblose Nadeln, welche sich schwer in Wasser und kaltem Alkohol, lejchter in heissem Alkohol lösen. In verdünnten Säuren löst sich der Körper äusserst leicht unter Bildung von Salzen auf, welche auch krystallinisch erhalten werden können. Der Schmelzpunkt liegt bei $188^{\circ}$.

Analysen:

I. 0,1596 Substanz gaben $0,3028 \quad \mathrm{CO}_{2}=0,08258 \mathrm{C}=51,74 \% \mathrm{C}$ und $0,0863 \mathrm{H}_{2} \mathrm{O}=0,00959 \mathrm{H}=6,00 \stackrel{\circ}{\circ} \mathrm{H}$.

II. 0,1154 Substanz gaben liei $21^{\circ}$ und $740 \mathrm{~mm}$ Druck $28,1 \mathrm{ccm}$ feuchten $N=0,03128 \mathrm{~N}=27,10 \% \mathrm{~N}$. 
G. Frerichs u. H. Beckurts: Phenylhydrazidoharnstoffe.

Berechnet für die Formel

$$
\begin{gathered}
\mathrm{C}_{9} \mathrm{H}_{12} \mathrm{~N}_{4} \mathrm{O}_{2}: \\
\mathrm{C}_{9}=108=51,92 \% \\
\mathrm{H}_{12}=12=5,77 \% \\
\mathrm{~N}_{1}=56=26,92 \% \\
\mathrm{O}_{2}=32=15,39 " \\
\hline 208=100,00 \% .
\end{gathered}
$$

Gefunden :

$$
\text { I. }
$$

$51,74 \% \quad-$

$6,00, \quad-$

Die Benzaldehydverbindung des a-Phenylhydrazidoacetylharnstoffes $\mathrm{C}_{16} \mathrm{H}_{16} \mathrm{~N}_{4} \mathrm{O}_{2}$ wurde aus der mit Benzaldehyd kurze Zeit erwärmten alkoholischen Lösung des Phenylhydrazidoacetylharnstoffes in farblosen Nadeln erhalten, welche bei $219^{\circ}$ schmolzen.

Analyse:

0,1014 Substanz gaben $0,2422 \mathrm{CO}_{3}=0,06605 \mathrm{C}=65,13_{, 3}^{\circ} \mathrm{C}$ und $0,0518 \mathrm{H}_{8} \mathrm{O}=0,00575 \mathrm{H}=5,67 \%$.

Die Formel $\mathrm{C}_{16} \mathrm{H}_{10} \mathrm{~N}_{4} \mathrm{O}_{2}$ verlangt $64,86 \% \mathrm{C}$ und $5,07 \% \mathrm{H}$.

\section{a-Phenylhydrazidoacetylmethylharnstoff}<smiles>CNC(=O)Nc1ccccc1</smiles>

Aus Chloracetylmethylharnstoff und Phenylhydrazin dargestellt, gleicht derselbe in Krystallform, Löslichkeit dem entsprechenden Derivat des Harnstoffes. Der Schmelzpunkt liegt bei $185^{\circ}$.

\section{Analysen:}

I. 0,1621 Substanz gaben $0,3202 \mathrm{CO}_{2}=0,08732 \mathrm{C}=033,87 \%$ C and $0,0951 \mathrm{H}_{2} \mathrm{O}=0,01056 \mathrm{H}=6,51 \% \mathrm{H}$.

II. 0,1225 Substanz gaben bei $25^{\circ}$ und $741 \mathrm{~mm}$ Druck $28,7 \mathrm{ccm}$ feuchten $\mathrm{N}=0,03119 \mathrm{~N}=25,46 \% \mathrm{~N}$.

Berechnet für die Formel

$$
\begin{gathered}
\mathrm{C}_{10} \mathrm{H}_{14} \mathrm{~N}_{4} \mathrm{O}_{2}: \\
\mathrm{C}_{10}=120=54,05 \% \\
\mathrm{H}_{14}=14=6,30 \% \\
\mathrm{~N}_{4}=56=25,22 \% \\
\mathrm{O}_{2}=32=14,41 \% \\
\hline 222=99,98 \% .
\end{gathered}
$$

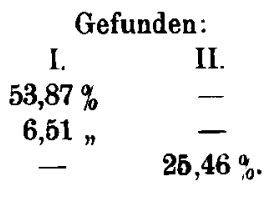

Die Benzaldehydverbindung des a - Phenylhydrazidoacetylmethylharnstoffes $\mathrm{C}_{17} \mathrm{H}_{18} \mathrm{~N}_{4} \mathrm{O}_{2}$ wurde wie die entsprechende Verbindung des Harnstoffes. dargestellt. Sie bildet farblose Nadeln, welche bei $238^{\circ}$ schmelzen. 


\section{Analyse:}

0,1242 Substanz gaben bei $22^{\circ}$ und $770 \mathrm{~mm}$ Druek $19,5 \mathrm{ccm}$ feuchten $\mathrm{N}=0,02238 \mathrm{~N}=18,01 \% \mathrm{~N}$.

Die Formel $\mathrm{C}_{17} \mathrm{H}_{18} \mathrm{~N}_{4} \mathrm{O}_{2}$ verlangt $17,76 \% \mathrm{~N}$.

$$
\begin{aligned}
& \text { a. Phenylhydrazidoacetylphenylharnstoff } \\
& \mathrm{CO}\left\langle\frac{\mathrm{NH} \mathrm{CO} \mathrm{CH} \mathrm{CH}_{2}}{\mathrm{NC}_{6} \mathrm{H}_{5}}{\stackrel{\mathrm{NH}}{\mathrm{N}_{2}}}_{\mathrm{NH}}=\mathrm{C}_{15} \mathrm{H}_{16} \mathrm{~N}_{4} \mathrm{O}_{2}\right. \text {. }
\end{aligned}
$$

Derselbe bildet farblose Nadeln, welche bei $180^{\circ}$ schmelzen. In Alkohol und namentlich in verdünnten Säuren löst er sich schwerer auf, wie die oben beschriebenen Derivate des Harnstoffes und Methylharnstoffes.

$$
\text { Analysen: }
$$

I. 0,1254 Substanz gaben bei $21^{\circ}$ und $753 \mathrm{~mm}$ Druck $22,2 \mathrm{ccm}$ feuchten $\mathrm{N}=0,02503 \mathrm{~N}=19,96 \% \mathrm{~N}$.

II. 0,1096 Substanz gaben bei $21^{\circ}$ und $757 \mathrm{~mm}$ Druck $18,9 \mathrm{ccm}$ feuchten $\mathrm{N}=0,02 ! 43 \mathrm{~N}=19,55 \% \mathrm{~N}$.

III. 0,1489 Substanz gaben $0,3476 \mathrm{CO}_{2}=0,0948 \mathrm{C}=63,66^{\circ}{ }_{0} \mathrm{C}$ and $0,0766 \mathrm{H}_{2} \mathrm{O}=0,00851 \mathrm{H}=5,71 \% \mathrm{H}$.

Berechnet für die Formel

$$
\begin{array}{cccc}
\mathrm{C}_{16} \mathrm{H}_{18} \mathrm{~N}_{4} \mathrm{O}_{2}: & \text { I. } & \text { II. } & \text { III. } \\
\mathrm{C}_{16}=180=63,38 \% & - & - & 63,66 \% \\
\mathrm{H}_{16}=16=5,63 \% & - & - & 5,71 \% \\
\mathrm{~N}_{4}=56=19,71 \% & 19,96 \% & 19,55 \% & - \\
\mathrm{O}_{2}=32=11,27 \% & & &
\end{array}
$$

Gefunden:

Die Benzaldehydverbindung des a-Phenylhydrazidoacetylphenylharnstoffes $\mathrm{C}_{22} \mathrm{H}_{20} \mathrm{~N}_{4} \mathrm{O}_{2}$ bildet kleine, farblose Nädelchen, welche bei $177^{\circ}$ schmelzen.

$$
\begin{aligned}
& \text { a-Phenylhydrazidoacetyl-p -äthoxylphenylharnstoff } \\
& \qquad \mathrm{CO}\left\langle\begin{array}{ll}
\mathrm{NH} \mathrm{C}_{6} \mathrm{H}_{4} \mathrm{OC}_{2} \mathrm{H}_{5} \mathrm{NH}_{2} \\
\mathrm{NH} \mathrm{CO} \mathrm{CH}_{2} & \mathrm{NC}_{6} \mathrm{H}_{5}
\end{array}=\mathrm{C}_{17} \mathrm{H}_{20} \mathrm{~N}_{4} \mathrm{O}_{3}\right. \text {. }
\end{aligned}
$$

Derselbe bildet weiche, filzige Nadeln, welche bei $169^{\circ}$ schmelzen.

$$
\text { Analyse: }
$$

0,1024 Substanz gaben $0,2320 \mathrm{CO}_{2}=0,06327 \mathrm{C}=61,78 \% \mathrm{C}$ und $0,0590 \mathrm{H}_{2} \mathrm{O}=0,006555 \mathrm{H}=6,40 \% \mathrm{H}$.

Die Formel $\mathrm{C}_{17} \mathrm{H}_{20} \mathrm{~N}_{4} \mathrm{O}_{3}$ verlangt $62,19 \% \mathrm{C}$ und $6,09 \% \mathrm{H}$. 
Die Benzaldehydverbindung des a - Phenylbydrazidoacetyl - päthoxylphenylharnstoffes $\mathrm{C}_{24} \mathrm{H}_{24} \mathrm{~N}_{4} \mathrm{O}_{3}$ bildet glänzende, farblose Nadeln, welche bei $179^{\circ}$ schmelyen.

Analyse:

0,1064 Substanz gaben $0,2668 \quad \mathrm{CO}_{2}=0,07276 \mathrm{C}=68,38 \% \quad \mathrm{C}$ und $0,0584 \mathrm{H}_{8} \mathrm{O}=0,006488 \mathrm{H}=6,09{ }_{1}^{\circ} \mathrm{H}$.

Die Formel $\mathrm{C}_{24} \mathrm{H}_{24} \mathrm{~N}_{4} \mathrm{O}_{3}$ verlangt $69,23 \% \mathrm{C}$ und $5,76 \% \mathrm{H}$.

Zur Darstellung der freien Phenylhydrazidoessigsäure wurden einige Gramm des Phenylhydrazidoacetylharnstoffes mit Natronlauge erwärmt. Es trat zunăchst klare Lösung ein und nach kurzer Zeit schieden sich reichliche Mengen von Krystallen ab, die jedenfalls das Natriumsalz der Phenylhydrazidoessigsäure darstellten. Gleichzeitig entwich viel Ammoniak. Nach dem Erkalten wurde mit. Salzsäure angesäuert und mit Aether ausgeschüttelt. Da aber dej Aether beim Verdunsten fast keinen Rückstand hinterliess, wurde die Flüssigkeit mit Natriumkarhonat wieder alkalisch gemacht und nun wieder vorsichtig Salzsäure zugesetzt, wodurch ein krystallinischer Niederschlag entstand, welcher abfiltriert und aus heissem Wasser umkrystallisiert wurde. Es resultierten auf diese Weise grosse glänzende Blättchen, welche sich bald etwas bräunten und bei $167^{\circ}$ unter starkem Aufschïumen schmolzen. Diese Thatsache liess mit Sicherheit vermuten, dass der erhaltene Körper nichts anderes als die asymmetrische Phenylhydrazidoessigsäure

$$
\mathrm{C}_{6} \mathrm{H}_{5}-\underset{\mathrm{NH}_{2}}{\mathrm{NCH}_{2} \mathrm{COOH}}
$$

darstellte, da sowohl der Schmelzpunkt wie auch die übrigen Eigenschaften genau mit den von C. D. Harries ${ }^{1}$ ) über diese Säure gemachten Angaben übereinstimmen. Die Analyse der Säure ergab folgende Zahlen:

0,1306 Substanz gaben $0,2754 \quad \mathrm{CO}_{2}=0,0751 \mathrm{C}=57,50 \% \mathrm{C}$ und $0,0706 \mathrm{H}_{2} \mathrm{O}=0,007844 \mathrm{H}=6,00 \stackrel{\%}{\%} \mathrm{H}$.

Die Formel der a-Phenylhydrazidoessigsäure $\mathrm{C}_{8} \mathrm{H}_{10} \mathrm{~N}_{2} \mathrm{O}_{2}$ verlangt $57,83 \% \mathrm{C}$ und $6,02 \% \mathrm{H}$.

Durch diesen Versuch war der Beweis geliefert, dass durch die Einwirkung von Phenylhydrazin auf Chloracetylharnstoffe neben anderen Verbindungen auch die Harnstoffderivate der a-Phenylhydrazidoessigsäure gebildet werden.

Es wurden nun zunächst die eingangs als I. Produkt bezeichneten Verbindungen, welche aus Chloracetylphenylharnstoff und atus p-Aethoxyl-

1) Ber. 28, 1226. 
phenylharnstoff mit Phenylhydrazin erhaiten waren, untersucht. Beide Körper behielten auch nach mehrmaligem Umkrystallisieren eine rein gelbe Farbe. Die Analyse der Verbindung des Phenylharnstottes ergab folgende Zahlen:

I. 0,1460 Substanz gaben $0,3406 \mathrm{CO}_{2}=0,09289 \mathrm{C}=63,62{ }_{o}^{\circ} \mathrm{C}$ und $0,0674 \mathrm{H}_{2} \mathrm{O}=0,00749 \mathrm{H}=\overline{0}, 13 \% \mathrm{H}$.

II. 0,1194 Substanz gaben bei $24^{\circ}$ und $751 \mathrm{~mm}$ Druck $21,5 \mathrm{ccm}$ feuchten $\mathrm{N}=0,02382 \mathrm{~N}=19,94 \% \mathrm{~N}$.

Der gefundenen Zusammensetzung entspricht die Formel $\mathrm{C}_{15} \mathrm{H}_{14}$ $\mathrm{N}_{4} \mathrm{O}_{2}$, welche $63,82 \% \mathrm{C}, 4,96^{\circ} / \mathrm{H}$ und $19,85 \% \mathrm{~N}$ verlangt.

Fast ebenso gut würden die gefundenen Zahlen bei Annahme der Formel eines symmetrischen Phenylhydrazidoacetylphenylharnstoffes

stimmen.

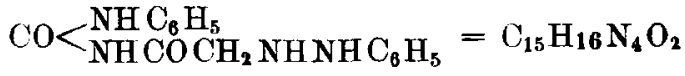

Diese Formel verlangt $63,38 \% \mathrm{C}, 19,71 \% \mathrm{~N}$, aber $5,63 \% \mathrm{H}$, während nur 5,13\% $\mathrm{H}$ gefunden wurden. $\mathrm{Da}$ nun ausserdem in den meisten Fällen die Analyse etwas zu hohe Zahlen für Wasserstoff er. giebt, so verdient die erstere Formel $\mathrm{C}_{15} \mathrm{H}_{14} \mathrm{~N}_{4} \mathrm{O}_{2}$ mit 4,96\% $\mathrm{H}$ den Vorzug.

Ganz analoge Zusammensetzung ergab die Analyse für den aus Chloracetyl-p-äthoxylphenylharnstoff erhaltenen Körper.

I. 0,1975 Substanz gaben $0,4504 \mathrm{CO}_{2}=0,1228 \mathrm{C}=62,17 \% \mathrm{C}$ und $0,1054 \mathrm{H}_{2} \mathrm{O}=0,01171 \mathrm{H}=5,93 \% \mathrm{H}$.

II. 0,1192 Substanz gaben bei $20^{\circ}$ und $750 \mathrm{~mm}$ Druck $18,2 \mathrm{ccm}$ feuchten $\mathbf{N}=0,02054 \mathrm{~N}=17,23 \% \mathrm{~N}$.

Die Formel $\mathrm{C}_{17} \mathrm{H}_{18} \mathrm{~N}_{4} \mathrm{O}_{8}$ verlangt $62,57 \% \mathrm{C}, 5,52 \% \mathrm{H}$ und 17,23\% $\mathrm{N}$.

Die um $2 \mathrm{H}$-Atome reichere Formel $\mathrm{C}_{17} \mathrm{H}_{20} \mathrm{~N}_{4} \mathrm{O}_{3}$ verlangt dagegen $62,19 \% \mathrm{C}, 17,07 \% \mathrm{~N}$ and $6,09 \% \mathrm{H}$.

Auch hier hat also die wasserstoffärmere Formel die grössere Wahrscheinlichkeit für sich.

Nun existiert eine Verbindung, welche 2 Atome Wasserstoff weniger besitzt als die symmetrische Phenylhydrazidoessigsäure. Es ist dieses die Phenylhydrazidoglyoxylsäure, welche die Formel

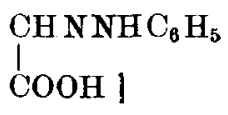

besitzt und aus der symmetrischen Phenylhydrazidoessigsäure sehr leicht durch Oxydation entsteht. ') Diese Säure stellt g e l be Nadeln dar, hierdurch

1) Ber. 28, 1230. 
würde also die gelbe Farbe der beiden Verbindungen erklärt werden. Leider genügte die zur Verfügung stehende Menge des Materiales nicht, durch Verseifung der Körper die freie Săure darzustellen, es ist aber mit Sicherheit anzunehmen, dass bei der Einwirkung von Phenylhydrazin auf Chloracetylphenylharnstoff und auf Chloracetyl-p-äthoxylphenylharnstoff ausser den Derivaten der asymmetrischen Phenylhydrazidoessigsăure auch die Derivate der symmetrischen Säure entstehen, welche aber durch spontane Oxydation in Derivate der Phenylhydrazinglyoxylsäure übergehen.

Phenylhydrazinglyoxylphenylharnstoff

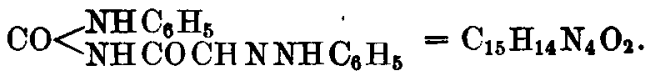

Derselbe stellt gelbe glänzende Nadeln dar, welche sich nicht in Wasser, schwer in kaltem Alkohol, leichter in heissem Alkohol and in Eisessig lösen und bei $197^{\circ}$ schmelzen.

\section{Phenylhydrazinglyoxylyl-p-äthoxylphenylharnstoff$$
\mathrm{CO}<\frac{\mathrm{NH} \mathrm{C}_{6} \mathrm{H}_{4} \mathrm{OC}_{2} \mathrm{H}_{5}}{\mathrm{NHCOHN} \mathrm{NH}_{6} \mathrm{H}_{5} .}
$$

Derselbe schmilzt bei $151^{\circ}$ und gleicht im tubrigen dem entsprechenden Derivat des Phenylharnstoffs. Beide Verbindungen reduzieren Fehling'sche Lösung erst nach längerem Erhitzen, worin sie ebenfalls mit der Phenylhydrazinglyoxylsäure übereinstimmen.

Es blieben nun noch die aus Chloracetylharnstoff, -methylharnstoff und Urethan erhaltenen, eingangs als I. Produkt bezeichneten Körper zu untersuchen tibrig. Das Derivat des Methylharnstoffes ging uns leider durch einen unglücklichen Zufall verloren und eine Neudarstellung war uns aus Mangel an Ausgangsmaterial bis jetzt noch nicht möglich. Die Untersuchung der beiden anderen Verbindungen ergab zunächst, dass beide bei derselben Temperatur, $275^{\circ}$, schmolzen, und dass auch ein Gemisch beider denselben Schmelzpunkt besass. Beide Körper waren also identisch.

Die Analyse ergab folgende Zahlen:

I. Aus Urethan: 0,1332 Substanz gaben $0,3170 \mathrm{CO}_{2}=0,08645 \mathrm{C}$ $=64,90 \% \mathrm{C}$ und $0,0572 \mathrm{H}_{2} \mathrm{O}=0,006355 \mathrm{H}=4,77 \% \mathrm{H}$.

II. 0,1174 Substanz gaben bei 250 und $741 \mathrm{~mm}$ Druck $27 \mathrm{ccm}$ feuchten $\mathrm{N}=0,02934 \mathrm{~N}=24,99 \% \mathrm{~N}$.

Arch. d. Pharm. CexXXVII. Bde. 5. Heft. 
IU. Aus Harnstoff: 0,1018 Substanz gaben bei 190 und $765 \mathrm{~mm}$ Druck $22,3 \mathrm{ccm}$ feuchten $\mathrm{N}=0,02581 \mathrm{~N}=25,35 \% \mathrm{~N}$.

Der für beide Körper gefundenen $\mathrm{Zu}$ sammensetzung entspricht eine Formel $\mathrm{C}_{15} \mathrm{H}_{18} \mathrm{~N}_{6} \mathrm{O}$.

Dieselbe verlangt:

$$
\begin{aligned}
& \mathrm{C}_{1 \mathrm{~s}}=180=64,51 \% \\
& \mathrm{H}_{1 \mathrm{1}}=13=4,66 \% \\
& \mathrm{~N}_{\mathrm{b}}=70=25,09 \% \\
& 0=16=5,74 \% \\
& \hline 279=100,00 \%
\end{aligned}
$$

Gefunden:

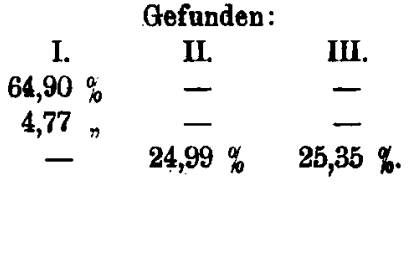

Die Bildụ̣g einer Verbindung von der angegebenen unitären Formel wird in folgender. Weise leicht erklärt:

Durch Einwirkang von Phenylhydrazin auf Acetylarethan hat A. Andreocci ${ }^{1}$ ) eine Verbindung von folgender Formel erhalten

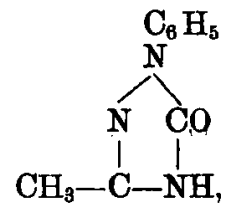

welche er als $\alpha$-Methyl- $\beta$-Phenylpyrrodiazolon bezeichnet. Dieser Körper bildet sich, indem das Phenylhydrazin mit der CO-Gruppe des Essigsãurerestes unter Wasserabspaltung reagiert, worauf unter Abspaltung von Alkohol Ringbildung eintritt. Aus dem Chloracetylurethan entsteht nun eine etwas kompliziertere Verbindung, indem zunächst, ähnlich wie beim Chloracetylphenylharnstoff, ein Derivat der symmetrischen Phenylhydrazidoessigsăure von der Formel

$$
\mathrm{CO}<\underset{\mathrm{NH} \mathrm{CO} \mathrm{CH}_{2}}{\mathrm{OCH}_{2} \mathrm{H}_{5}} \mathrm{NH} \mathrm{C}_{6} \mathrm{H}_{5}
$$

entsteht. Dieser Körper geht durch Oxydation sofort in das um $2 \mathrm{H}$ Atome ärmere Derivat der Phenylhydrazinglyoxylsäure

über.

$$
\mathrm{CO}<{ }_{\mathrm{NH}}^{\mathrm{OCO}_{2} \mathrm{CO}_{5}} \mathrm{CHN} \mathrm{NH} \mathrm{\textrm {C } _ { 6 } \mathrm { H } _ { 5 }}
$$

Diese Verbindung unterscheidet sich nun von der entsprechenden Verbindung des Phenylharnstoffes dadurch, dass sie eine leicht abspaltbare Alkoholpruppe enthält, während aus dem letzteren das entsprechende Anilin nicht leicht abspaltbar ist. Aus diesem Grunde reagiert die in der obigen Formel durch stärkeren Druck hervorgehobene CO-Gruppe

1) Ber. 22, R. 737. 
mit einem weiteren Molekul Phenylhydrazin, worauf durch Abspiitung von Alkohol Ringschliessung leicht eintritt, und ein Körper von folgender Formel entsteht,<smiles>CN1CNC(C=NNc2ccccc2)=NC1=O</smiles>

welcher die oben angefăhrte unitäre Formel $\mathrm{C}_{15} \mathrm{H}_{18} \mathrm{~N}_{3} \mathrm{O}$ besitzt.

Da aus dem Phenylhydrazinglyoxylphenylharnstoff das Anilin nicht so leicht abgespalten werden kann wie aus dem Urethanderivat der Alkohol, so unterbleibt hier die Einwirkang des Phenylhydrazins anf die COgruppe, da eine Ringbildung anch nach Eintritt des Phenylhydrazins nur sehr schwierig eintreten könnte.

Anders liegen dagegen die Verhältnisse beim gewöhnlichen Harnstoff. Hier tritt genau dieselbe Reaktion auf wie beim Urethan, da aus dem sich zunăchst bildenden Phenylhydrazinglyoxylderivat des Harnstoffes ebenso leicht Ammoniak, wie aus dem Urethanderivat Alkohol abgespalten wird. Es bildet sich in diesem Falle also derselbe Körper wie aus dem Urethan.

Die Verbindung $\mathrm{C}_{15} \mathrm{H}_{18} \mathrm{~N}_{5} \mathrm{O}$ stellt etwas gelblich gefärbte, bei $275^{\circ}$ schmelzende Nadeln dar, welche in Wasser, Alkohol und Eisessig in der Kalte sehr wenig, in den beiden letzteren in der Hitze etwas leichter löslich sind. Sie besitzt schwach săureartigen Charakter, löst sich in Laugen, wenn auch schwer und wird durch Sănren wieder ausgefällt. Durch Versetzen der ammoniakalischen Lösung mit Silbernitrat wurde eine Silberverbindung von der Formel $\mathrm{C}_{15} \mathrm{H}_{12} \mathrm{Ag} \mathrm{N}_{5} \mathrm{O}$ erhalten.

\section{Analyse:}

0,2790 Substanz gaben 0,0786 $\mathrm{Ag}=28,17 \% \mathrm{Ag}$, berechnet $27,98 \%$

Die analoge Silberverbindung von der Formel<smiles></smiles>

wurde von Andreocci dargestellt. 
Was nun die Mengenverhältnisse der bei der Einwirkung von Phenylhydrazin auf die Chloracetylharnstoffe entstehenden Körper betrifft, so ist folgendes zu bemerken: Beim gewöhnlichen Harnstoff sowie beim Methylharnstoff entstehen vorwiegend die Derivate der a-Phenylhydrazidoessigsäure, beim Phenyl-undp-Aethoxylphenylharnstoff entsteht fast ebensoviel von den Derivaten der Phenylhydrazinglyoxylsäure wie der a-Phenylhydrazidoessigsäure. Diese Thatsache steht in guter Uebereinstimmung mit der eingangs erwähnten Erscheinung, dass bei Verbindungen des Chloressigsäurerestes mit Radikalen saurer Natur die symmetrischen, mit Radikalen basischer Natur die asymmetrischen Verbindungen entstehen. In den Harnstoffen tritt eben neben dem basischen Charakter auch noch der Säurecharakter hervor und zwar umsomehr, je mehr andere Radikale nicht basischer Natur wie $\mathrm{C}_{6} \mathrm{H}_{5}$, $\mathrm{C}_{6} \mathrm{H}_{4} \mathrm{OC}_{2} \mathrm{H}_{5} \mathrm{im}$ Harnstoff enthalten sind. Aus diesem Grunde wurde auch ein asymmetrisches Derivat des Urethans nicht erhalten, weil im Urethan der basische Charakter durch die Gruppe $\mathrm{OC}_{2} \mathrm{H}_{5}$ wieder aufgehoben wird. Weitere Aufklärung über den Verlauf der verschiedenen Reaktionen hoffen wir durch eingehende Untersuchung der oben erwähnten gelbgefärbten, amorphen Körper zu erhalten.

Im Anschluss hieran möchten wir die Untersuchung eines Zersetzungsproduktes des a-Phenylhydrazidoacetylharnstoffes erwähnen.

Wie wir nachgewiesen haben ${ }^{1}$ ), entstehen aus den aromatischen Glycinylderivaten des Harnstoffes sehr leicht durch Abspaltung von Ammoniak aromatische $\beta$-Hydantoine, also ringförmige Verbindungen von der Formel<smiles>[R]N1CC(=O)NC1=O</smiles>

In ganz analoger Weise konnte nun aus dem Phenylhydrazidoacetylharnstoff eine ebenfalls ringförmige Verbindung entstehen, welche sich vom Phenylhydantoin dadurch unterschied, dass sie eine NH-Gruppe im Ringe mehr enthielt. Ein Versuch bestätigte die Richtigkeit dieser Voraussetzung.

Einige Gramm des a-Phenylhydrazidoacetylharnstoffes wurden in einem Kölbchen im Paraffinbade bis etwa $200^{\circ}$ erhitzt, bis die auftretende, lebhafte Ammoniakentwickelung beendet war. Nach dem Erkalten war der Inhalt des Kölbchens zu einer harten, glasigen Masse erstarrt. Dieselbe wurde zunächst mit heissem Alkohol behandelt, worin sie aber nur zum Teil sich auflöste. Durch Umkrystallisieren

1) S. oben. 
aus Eisessig wurde der in Alkohol sehr schwer lösliche Körper in kleinen, harten Oktaedern erhalten, welche bei $229^{\circ}$ schmolzen.

\section{Analysen:}

I. 0,1157 Substanz gaben bei $20^{\circ}$ und $751 \mathrm{~mm}$ Druck $22,4 \mathrm{ccm}$ feuchten $\mathrm{N}=0,02532 \mathrm{~N}=21,88 \% \mathrm{~N}$.

II. 0,1585 Substanz gaben $0,3282 \mathrm{CO}_{2}=0,0895 \mathrm{C}=56,46 \% \mathrm{C}$ und $0,0706 \mathrm{H}_{2} \mathrm{O}=0,007844 \mathrm{H}=4,94 \% \mathrm{H}$.

Berechnet für die Formel

$$
\begin{array}{ccc}
\mathrm{C}_{9} \mathrm{H}_{9} \mathrm{~N}_{8} \mathrm{O}_{2}: & \text { I. } & \text { II. } \\
\mathrm{C}_{9}=108=56,54 \% & - & 56,46 \% \\
\mathrm{H}_{9}=9=4,71 \% & - & 4,94 \% \\
\mathrm{~N}_{8}=42=21,98 \% & 21,88 \% & - \\
\mathrm{O}_{2}=32=16,75 \% & &
\end{array}
$$

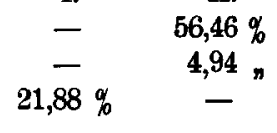

Gefunden:

Der unitären Formel $\mathrm{C}_{9} \mathrm{H}_{9} \mathrm{~N}_{8} \mathrm{O}_{2}$ entspricht ein Körper von folgender Konstitution:<smiles>CCCCCCCCC(=O)NC(=O)O</smiles>

welcher aus dem a-Phenylhydrazidoacetylharnstoff durch Abspaltung von Ammoniak nach folgendem Schema entsteht:

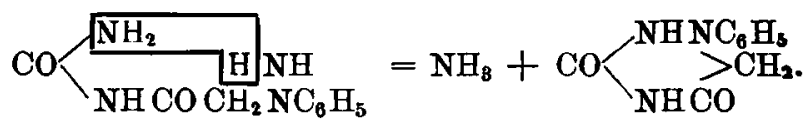

Wie man das $\beta$-Phenylhydantoin als Glykolylphenylharnstoff auffassen kann, so lässt sich die neue Verbindung als Glykolylphenylsemikarbazid bezeichnen. Nach der für stickstoffhaltige heterocyklische Verbindungen eingeführten Nomenklatur möchten wir aber den Körper als ein Triazinderivat, als $n$-Phenyldiketotetrahydro$\alpha$-triazin auffassen. Als solches gehört dieser Körper in dieselbe Klasse wie das 1-, 2-Diphenyl-3-oxy-a-triazin

$$
\begin{aligned}
& \mathrm{C}_{6} \mathrm{H}_{5} \mathrm{C}=\mathrm{N}-\mathrm{COH} \\
& \mathrm{C}_{6} \mathrm{H}_{5} \mathrm{C}=\mathrm{N}-\mathrm{N},
\end{aligned}
$$

welches ans Benzil und Semikarbazid ${ }^{1}$ ) erhalten wurde und die n-Phenyl- und n-Phenyl-n-alkylketotetrahydro- $\alpha$-triazine,<smiles>[R][C@@H]1NCCON1</smiles>

ans $\alpha$-Phenylhydrazidoessigester und Formamid oder substituierten 
Formamiden. ${ }^{1}$ ) Der neue Körper entsteht ebenso leicht aus dem a-Pluenylhydrazidoacetylmethylharnstoff durch Erhitzen, wodurch in diesem Falle Methylamin abgespaiten wird.

Der Körper besitzt ähnlich wie die Hydantoine säureartigen tharakter und verbindet sich mit Alkalien. Durch Säuren wird er ans diesen Verbindnngen unverăndert wieder abgeschieden.

Ueber weitere Untersuchungen von Harnstoff- und Urethanderivaten werden wir später berichten.

\title{
Mitteilung aus der pharmazeutisehen Abteilung des chemischen Institutes der Königlichen Akademie Münsteri/W. Von G. Kassner.
}

\section{Jeber das fette Oel des Quittensamens ${ }^{2}$ ).}

\author{
Von Robert Herrmann.
}

(Eingegangen den 25. V. 1899.)

Ueber die Inhaltsstoffe der Samen von Cydonia vulgaris existieren in der Litteratur nur dürftige Angaben. Vor allem fehlt es an Nachrichten über die chemische Beschaffenheit des neben $13 \%$ Aschenbestandteilen, 22\% Pflanzenschleim, ferner Amygdalin, Gerbstoff und Proteïnstoffen in den Samen enthaltenen fetten Oeles.

Auf Veranlassung des Professor Kassner, Dirigenten der pharm. Abteilung des chem. Instituts zu Münster, unternahm es der Verfasser, sowohl den Gehalt der Sem. Cydoniae an fettem Oel zu ermitteln, als auch die Zusammensetzung desselben zu studieren.

Der von der Firma Caesar \& Loretz in Halle bezogene Samen wurde fein gemahlen und nach vorsichtigem Trocknen der Extraktion mit gereinigtem Aether unterworfen. $\mathrm{Zu}$ einigen Versuchen wurde auch Chloroform und Petroläther mit Erfolg als Extraktionsmittel benutzt; letzterer gab sogar ein weniger gefärbtes Oel. Das Lösungsmittel wurde alsdann durch Erhitzen im Wasserbade abdestilliert; das zurückbleibende Oel filtriert und getrocknet.

1) Ber. 28, 1228.

2) Aus der gleichlautend betitelten Inaugural-Dissertation von Robert Herrmann, vorgelegt der philosophischen Fakultät der Friedrich-AlexanderUnirersität Erlangen, 1899. 